\title{
Clinical Evaluation of Novel Natural Polysaccharides Sacran as a Skincare Material for Atopic Dermatitis Patients
}

\author{
Satoshi Fukushima ${ }^{1^{*}}$, Keiichi Motoyama ${ }^{2}$, Yuki Tanida ${ }^{2}$, Taishi Higashi², Yoichi Ishitsuka ${ }^{2}$, \\ Yuki Kondo ${ }^{2}$, Tetsumi Irie ${ }^{2,3}$, Takumi Tanaka ${ }^{4}$, Hironobu Ihn"\#, Hidetoshi Arima ${ }^{2,3 \#}$ \\ ${ }^{1}$ Department of Dermatology and Plastic Surgery, Faculty of Life Sciences, Kumamoto University, Kumamoto, \\ Japan \\ ${ }^{2}$ Graduate School of Pharmaceutical Sciences, Kumamoto University, Kumamoto, Japan \\ ${ }^{3}$ Program for Leading Graduate Schools "HIGO (Health Life Science: Interdisciplinary and Glocal Oriented) \\ Program", Kumamoto University, Kumamoto, Japan \\ ${ }^{4}$ Daito Kasei Kogyo Co., Ltd., Osaka, Japan \\ Email: \#ihn-der@kumamoto-u.ac.jp,"\#arimah@gpo.kumamoto-u.ac.jp
}

Received 24 December 2015; accepted 28 February 2016; published 2 March 2016

Copyright $@ 2016$ by authors and Scientific Research Publishing Inc.

This work is licensed under the Creative Commons Attribution International License (CC BY).

http://creativecommons.org/licenses/by/4.0/

(c) () Open Access

\section{Abstract}

Atopic dermatitis is a skin disease characterized by inflammation, pruritus, and chronic or relapsing eczematous lesions. Recently, ampholytic polysaccharide sacran has attracted a particular focus of attention as a novel biomaterial. In the present study, we investigated the effect of sacran solution on atopic dermatitis in the clinical study. Almost all of the average scores for atopic dermatitis symptoms of each patient treated with sacran solutions were improved. In addition, the scores of sleep disorder and itching were also significantly ameliorated by the sacran treatment for 4 weeks, compared with those of initial states. In immatured dermal skin model stimulated with 2,4-dinitrofluorobenzene (DNFB), the sacran treatment markedly down-regulated inflammatory cytokine and chemokine mRNA levels such as $M C P-1, T N F-\alpha, I L-1 \beta$, and $I L-6$ mRNAs, compared with that of DNFB alone. Furthermore, a sacran solution significantly suppressed the mRNA expression of TNF- $\alpha$ and COX-2 in RAW264.7 cells, a murine macrophage-like cell line, stimulated with phorbol-12-myristate-13-acetate (PMA). In RBL-2H3 cells, a rat basophilic leukemia cell line, a sacran solution significantly lowered $\beta$-hexosaminidase release, indicating the suppression of allergic response. These results suggest that a sacran solution may have the potential to improve atopic dermatitis through the impairment of production of inflammatory cytokine and chemokine mRNA.

\footnotetext{
Equal contribution.

"Corresponding authors.
}

How to cite this paper: Fukushima, S., et al. (2016) Clinical Evaluation of Novel Natural Polysaccharides Sacran as a Skincare Material for Atopic Dermatitis Patients. Journal of Cosmetics, Dermatological Sciences and Applications, 6, 9-18. 


\section{Keywords}

\section{Sacran, Polysaccharide, Clinical Trial, Atopic Dermatitis, Skin Care}

\section{Introduction}

Atopic dermatitis is a skin disease characterized by inflammation, pruritus, and chronic or relapsing eczematous lesions. Atopic dermatitis negatively affects quality of life for both patients with atopic dermatitis as well as their caregivers. Management strategies for atopic dermatitis focus on maintaining the skin barrier and are recommended by medical societies worldwide. Use of mild, appropriately formulated emollients may provide benefits without interfering with skin barrier function. However, emollients alone may not control eczema or aspects of this skin disorder, especially in severe cases. Therefore, anti-inflammatory and immunomodulatory therapies may be necessary for moderate-to-severe atopic dermatitis until symptom resolution in skin (e.g., lesions, patches of dryness, or areas that are prone to flare). Nowadays, corticosteroids are commonly administered to reduce the inflammation. However, they can frequently cause a set of serious adverse effects [1] [2]. Hence, the great efforts have been devoted towards the discovery of new and safe anti-inflammatory drugs.

Recently, numerous beneficial effects of natural polysaccharides have been demonstrated on human health to exhibit a spectrum of biological activities such as antioxidant, antitumor, and anti-inflammatory [3]-[5]. For example, the heparan sulfate on the surface of all adherent cells modulates the actions of a large number of extracellular ligands [6]. Kawashima et al. reported that oversulfated chondroitin/dermatan sulfate chains are important in selectin- and/or chemokine-mediated cellular responses [7]. In addition, hyaluronan alternatively participates in leukocyte recruitment via interaction with CD44, while activating various inflammatory cells, such as macrophages, through CD44-dependent signaling [8]. Therefore, the field of polysaccharide biology provides new clues and explanations of the process of inflammation and suggests new therapeutic approaches to human disease.

Recently, ampholytic polysaccharide sacran has attracted a particular focus of attention. It is extracted from the Japanese indigenous cyanobacterium Aphanothece sacrum, which is mass-aquacultured in rivers with a high ionic concentration and which possesses a plenty of a jelly-like extracellular matrix with high water content $(97.5 \%$ - 98.3\%) [9]-[13]. Sacran is a heteropolysaccharide composed of various sugar residues such as glucose, galactose, mannose, xylose, rhamnose, fucose, galacturonic acid and glucuronic acid, and contains traces of alanine, galactosamine and muramic acid; $11 \%$ of the monosaccharides contain a sulfate group and $22 \%$ of them contain a carboxyl group. In addition, sacran was reported to be a supergiant molecule with extremely high molecular weight $\left(2.4 \times 10^{7} \mathrm{~g} / \mathrm{mol}\right)$ and surprising length (more than $\left.8 \mu \mathrm{m}\right)$. The safety of sacran as a biomaterial could be attributed to the long term usage of Aphanothece sacrum by inhabitants of the Kyushu region in Japan as a functional food to ameliorate allergic tendency and gastroenteritis. Recently, Ngatu et al. revealed that sacran has anti-inflammatory effects on 2,4,6-trinitrochlorobenzene-induced atopic dermatitis model mice [14]. However, there is no report on the application of sacran for human atopic dermatitis patients. Therefore, in the present study, we investigated the effect of sacran solution on atopic dermatitis in the clinical study.

\section{Materials and Methods}

\subsection{Materials}

Sacran was extracted from cyanobacterium Aphanothece sacrum, and was gifted by Daito Kasei Kogyo Co., Ltd. (Osaka, Japan). RPMI-1640 medium was purchased from Nissui Pharmaceuticals (Tokyo, Japan). TRIzol ${ }^{\circledR}$ Reagent and fatal bovine serum (FBS) were purchased from Invitrogen (Tokyo, Japan). 2,4-Dinitrofluorobenzene (DNFB) was purchased from Wako Pure Chemical Industries (Osaka, Japan). Monoclonal anti-dinitrophenyl IgE (Anti-DNP-IgE) and dinitrophenyl-bovine serum albumin (DNP-BSA) protein were purchased from Cosmo Bio (Tokyo, Japan). Phorbol-12-myristate-13-acetate (PMA) was purchased from Sigma Aldrich (Tokyo, Japan). All other chemicals and solvents were of analytical reagent grade.

\subsection{Extraction of Sacran}

Sacran was extracted from cyanobacterium Aphanothece sacrum followed by previous report [15]. Briefly, the 
Aphanothece sacrum (1 kg) was freeze-thawed, washed by distilled water to remove aqueous pigment (phycobilin), immersed and stirred in a water bath filled with a large amount of dilute hydrochloric acid ( $\mathrm{pH} 3$ ) at $60^{\circ} \mathrm{C}$ for $24 \mathrm{~h}$. After washed with 50\% - 70\% (v/v) isopropanol overnight, a polysaccharide was collected by filtration and air dried at $60^{\circ} \mathrm{C}$. The polysaccharide was extracted using $0.1 \mathrm{M}$ sodium hydroxide solution at $70^{\circ} \mathrm{C}$ with agitation for $3 \mathrm{~h}$. Then, the polysaccharides solution was adjusted to a $\mathrm{pH} 7$ to 8 by $0.1 \mathrm{M}$ hydrochloride and filtered by sterile cotton gauge. The filtrate was slowly poured into absolute isopropanol to precipitate white fibrous materials, which were then redissolved in hot water and reprecipitated 3 times. The polysaccharide fibers were dried (yield, $0.65 \mathrm{wt} \%$ - $0.75 \mathrm{wt} \%$ to wet biomaterials and $60 \mathrm{wt} \%$ - $70 \mathrm{wt} \%$ to dried ones). The aqueous solution showed no specific absorption in the wavelength range of 220 to $600 \mathrm{~nm}$ in UV-visible spectroscopy, which confirmed that there was no contamination by proteins, nucleic acid, chromospheres, and/or other chemicals with UV-visible absorption.

\subsection{Patients and Clinical Study Design}

This case descriptive, clinical and biochemical study was conducted in the Department of Dermatology and Plastic Surgery, Faculty of Life Sciences, Kumamoto University. Twenty five patients (5 males and 20 females) were enrolled in this study. The nature and aim of this study were explained for each patient. Formal consent was taken from each patient before this study. The clinical studies were approved by the Ethics Committee of Kumamoto University (Approval No.745). The patients used the sacran solution $(0.05 \%(\mathrm{w} / \mathrm{v}))$ for every morning and night after taking shower for 4 weeks. The patients enrolled in this study were well-controlled by standard therapy. During this clinical study, the standard therapy for patients did not changed at all. About 13 questionnaire items as indicated in Table 1, we obtained an answer from the patients with atopic dermatitis after treatment with sacran solution for 2 weeks and 4 weeks. Also, the dermatologists evaluated an area of disorder on face, total score of rash.

\subsection{Trans-Epidermal Water Loss (TEWL)}

Trans-epidermal water loss (TEWL) was determined by a VapoMeter Wireless SWL4 (Delfin technology, Finland). Before each measurement time, the apparatus will be calibrated according to the recommendations of the manufacturer. Measurements will be undertaken after a 20 min rest period, according to the guidelines set out by Standardization Group appointed by the European Society of Contact Dermatitis [16]. Room temperature will be kept between $20^{\circ} \mathrm{C}$ and $24^{\circ} \mathrm{C}$ and the relative humidity will be comprised between $40 \%(\mathrm{w} / \mathrm{v})$ and $60 \%(\mathrm{w} / \mathrm{v})$. Three acquisitions will be performed on the bony areas of the upper cheek of left and right sides.

\subsection{Cell Culture}

TESTSKIN ${ }^{\mathrm{TM}}$ LSE-high, a three-dimensional cultured skin, was purchased from TOYOBO Life Science (Osaka, Japan). RAW264.7 cells, a murine macrophage-like cell line, and RBL-2H3 cells, a rat basophilic leukemia cell

\begin{tabular}{l} 
Table 1. Scoring items. \\
Erythema \\
$\begin{array}{c}\text { Feeling with the shade of the skin } \\
\text { Itch of affected part inflammation }\end{array}$ \\
Irritation \\
Swelling of the affected part \\
$\begin{array}{c}\text { Desquamation of the affected part } \\
\text { Wound by the scratch, red swelling } \\
\text { Pain of affected part inflammation } \\
\text { Eyes inflammation } \\
\text { Affected part inflammation is gloomy } \\
\text { Moist feeling } \\
\text { A feeling of stickiness } \\
\text { Dry feeling of the skin }\end{array}$ \\
\hline
\end{tabular}


line, were obtained from Riken Bioresource Center (Tsukuba, Japan) and ATCC (Summit Pharmaceuticals International Corporation, Tokyo, Japan), respectively. RAW264.7 cells and RBL-2H3 cells were grown in RPMI1640 medium, containing $1 \times 10^{5} \mathrm{mU} / \mathrm{mL}$ of penicillin, $0.1 \mathrm{mg} / \mathrm{mL}$ of streptomycin supplemented with $10 \%$ FBS at $37^{\circ} \mathrm{C}$ in a humidified $5 \% \mathrm{CO}_{2}$ and $95 \%$ air atmosphere.

\subsection{Real Time Polymerase Chain Reaction (Real-Time PCR)}

Total RNA was isolated from TESTSKINTM or RAW264.7 cells after stimulation with DNFB or PMA, respectively, using TRIzol ${ }^{\circledR}$ Reagent according to the manufacturer's protocol. The RNA $(0.5 \mu \mathrm{g})$ was subsequently reverse-transcribed using ReverTra Ace ${ }^{\circledR}$ qPCR RT Master Mix (TOYOBO, Osaka, Japan). The expression levels of MCP-1, TNF- $\alpha$, IL-6, IL- $1 \beta$ and COX-2 were determined using real time PCR assay. Real-time PCR was performed on a CFX96 ${ }^{\mathrm{TM}}$ Real-Time PCR (BIO RAD, Tokyo, Japan) using $1 \mu \mathrm{L}$ of cDNA for each sample. THUNDERBIRD $^{\text {TM }}$ SYBR $^{\circledR}$ qPCR Mix (TOYOBO, Osaka, Japan) was used to detect products, and $10 \mu \mathrm{M}$ concentrations of the following primers were used: mouse GAPDH forward: 5'-GGTGAAGGTCGGTGTGAA CGGATT-3', mouse GAPDH reverse: 5'-AATGCCAAAGTTGTCATGGATGACC-3', MCP-1 forward: 5'-ATGCAATCAATGCCCCAGTC-3', MCP-1 reverse: 5'-TGCAGATTCTTGGGTTGTGG-3', mouse TNF- $\alpha$ forward: 5'-AACATCCAACCTTCCCAAACG-3', mouse TNF- $\alpha$ reverse: 5'-CTCTTAACCCCCGAACTCCC AG-3', mouse IL-6 forward:5'-TGGAGTCACAGAAGGAGTGGCTAAG-3', mouse IL-6 reverse: 5'-TCTGAC CACAGTGAGGAATGTCCAC-3', mouse IL-1 $\beta$ forward: 5'-AAACCTCTTCGAGGCACAAG-3', mouse IL-1 $\beta$ reverse: 5'-GTTTAGGGCCATCAGCTTCA-3', mouse COX-2 forward: 5'-AGCACTGCATCCTGC CAGCTC-3', mouse COX-2 reverse: 5'-AGAGGACTGTGGCTCTGACC-3'. The relative amount of cDNA in each sample was normalized using GAPDH, and the melting curve was used to verify specificity. PCR was set at $95^{\circ} \mathrm{C}$ initially for $30 \mathrm{~s}$, followed by 40 cycles of $95^{\circ} \mathrm{C} \times 15 \mathrm{~s}, 55^{\circ} \mathrm{C} \times 15 \mathrm{~s}, 72^{\circ} \mathrm{C} \times 45 \mathrm{~s}$. The relative amount of cDNA in each sample was normalized using GAPDH, and the melting curve was used to verify specificity.

\section{7. $\beta$-Hexosaminidase Release from RBL-2H3 Cells}

The release of $\beta$-hexosaminidase from RBL-2H3 cells were evaluated by the following modified method [17]. RBL-2H3 cells in 24 -wellplate $\left(5 \times 10^{5}\right.$ cells $\left./ \mathrm{mL}\right)$ were sensitized with anti-DNP IgE $(0.45 \mu \mathrm{g} / \mathrm{mL})$, and incubated for $24 \mathrm{~h}$. Then, the cells were washed with $400 \mu \mathrm{L}$ of Siraganian buffer (buffer A) [119 mM NaCl, $5 \mathrm{mM}$ $\mathrm{KCl}, 5.6 \mathrm{mM}$ glucose, $0.4 \mathrm{mM} \mathrm{MgCl}_{2}, 1 \mathrm{mM} \mathrm{CaCl}_{2}, 25 \mathrm{mM}$ piperazine- $N, N$ '-bis (2-ethanesulfonic acid) (PIPES), $0.1 \% \mathrm{BSA}$, and $40 \mathrm{mM} \mathrm{NaOH}, \mathrm{pH} 7.2]$. An aliquot of buffer A (160 $\mu \mathrm{L})$ was added and incubated for $10 \mathrm{~min}$ at $37^{\circ} \mathrm{C}$. The $20 \mu \mathrm{L}$ of test sample solution was added to each well and incubated for 10 min, followed by the addition of $20 \mu \mathrm{L}$ of antigen (DNP-BSA, final concentration $10 \mu \mathrm{g} / \mathrm{mL}$ ) at $37^{\circ} \mathrm{C}$ for 20 min to stimulate cell degranulation. The supernatants $(50 \mu \mathrm{L})$ were transferred into 96 well microplate and incubated with $50 \mu \mathrm{L}$ of substrate PNAG ( $1 \mathrm{mM}$ p-nitrophenyl- $N$-acetylb-D-glucosaminide) in $0.1 \mathrm{M}$ citrate buffer (pH 4.5 ) at $37^{\circ} \mathrm{C}$ for 3 h. The reaction was stopped by adding $200 \mu \mathrm{L}$ of stop solution $\left(0.1 \mathrm{M} \mathrm{Na}_{2} \mathrm{CO}_{3} / \mathrm{NaHCO}_{3}, \mathrm{pH} 10.0\right)$. The absorbance was measured with a microplate reader at $405 \mathrm{~nm}$. The percentage of $\beta$-hexosaminidase release into supernatant was calculated according to the following formula: $\beta$-Hexosaminidase release ratio $(\%)=100 \times \mathrm{S} /(\mathrm{S}+$ $\mathrm{CL}$ ), where CL and S are the absorbance of cell lysate and the absorbance of supernatant, respectively.

\subsection{Statistical Analysis}

In statistical analysis of grades in clinical studies evaluated by dermatologists, the $p$-value was determined employing the Wilcoxon signed-rank test, followed by the Bonferroni correction. In statistical analysis for in vitro data are presented as the mean \pm S.E. Significant differences between experimental values were determined using Scheffe's test, with $p<0.05$ considered significance.

\section{Results and Discussion}

\subsection{Clinical Study}

We analyzed the effects of sacran solution on the symptom of atopic dermatitis based on the 13 questionnaire results. The average scores more than 2 or less than 2 indicates the improvement or exacerbation of the symptoms of atopic dermatitis, respectively. As shown in Figure 1, almost all of the average scores of each patient treated with sacran solutions were more than 2 . In addition, the scores of sleep disorder and itching tended to 


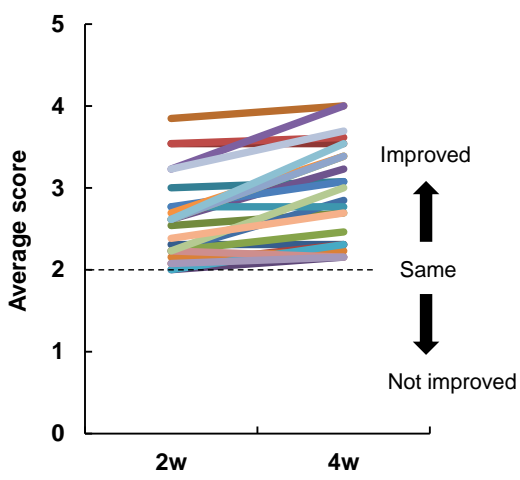

Figure 1. Effect of sacran solution on atopic dermatitis symptoms in patients. Sacran solution was applied for 4 weeks in 25 patients with atopic dermatitis. After 2 and 4 weeks, the patients treated with sacran solution judged the atopic dermatitis symptoms by themselves.

decrease during the sacran treatment for 4 weeks with significant differences compared those of initial states (0 week) (Figure 2). These results suggest that the patients judged amelioration of their atopic dermatitis by the treatment with sacran solution.

Based on the dermatologist analysis, the area of rash on face in atopic dermatitis patients were significantly decreased (Figure 3(a)). In addition, the total scores of rash were also lowered by the treatment with sacran solution for 4 weeks. Taken together, the sacran solution may have the potential to ameliorate the symptom of atopic dermatitis.

The dry itchy skin worsens the severity of atopic dermatitis and associated with the increase in scratching behavior [18]. Next, we therefore investigated the effects of topical application of sacran on TEWL in 10 humans with dry skin (Figure 4). As the results, sacran solution markedly suppressed TEWL, compared to hyaluronan, a well-known polysaccharide that is reported to improve skin moisture and provide skin barrier repair activity [19]. Thereby, these results suggest that sacran can ameliorate the skin barrier protection and repair in atopic dermatitis patients.

\subsection{Effects of Sacran Solution on mRNA Levels in Immatured Dermal Skin Model Stimulated with DNFB}

The itchy skin elicits scratching behavior, leading to mechanical skin injury and the release of various inflammatory cytokines or chemokines [20] [21]. Therefore, we examined the effect of sacran on inflammatory cytokine and chemokine mRNA levels in immatured dermal skin model stimulated with DNFB. Here, we used immatured dermal skin model (Toyobo, Co, Ltd, Osaka, Japan) simulated the skin of atopic dermatitis patients having low skin barrier function. DNFB was chemical antigen to evoke contact hypersensitivity reaction and was widely used as an inducer of atopic dermatitis. After treatment with DNFB and sacran for $24 \mathrm{~h}$, inflammatory cytokine and chemokine mRNA levels were determined by real-time PCR. As shown in Figure 5, MCP-1, $T N F-\alpha, I L-1 \beta$ and $I L-6$ mRNAs were significantly down-regulated by the treatment with sacran, compared to those of DNFB alone. These results suggest that sacran may have the potential to reduce inflammatory cytokine and chemokine mRNA levels in skin of atopic dermatitis.

To reveal the anti-inflammatory activity of sacran, it is extremely important to investigate whether a sacran penetrates skin or not. Therefore, we examined the skin penetration of sacran in matured and immatured dermal skin models after treatment for $24 \mathrm{~h}$. As a results, biotinylated sacran $(0.05 \%(\mathrm{w} / \mathrm{v}))$ was penetrate the dermal in immatured dermal skin model, but not in matured dermal skin model, simulated the skin of healthy human having rigid skin barrier function (data not shown). These results suggest that a sacran can permeate into the skin of atopic dermatitis with low skin barrier functions, but not into the healthy human skin.

\subsection{Effect of Sacran Solution on TNF- $\alpha$ and COX-2 mRNA Levels in Macrophages Stimulated with PMA}

PMA, a specific activator of protein kinase C (PKC), induces suitable inflammatory responses. Next, to examine 


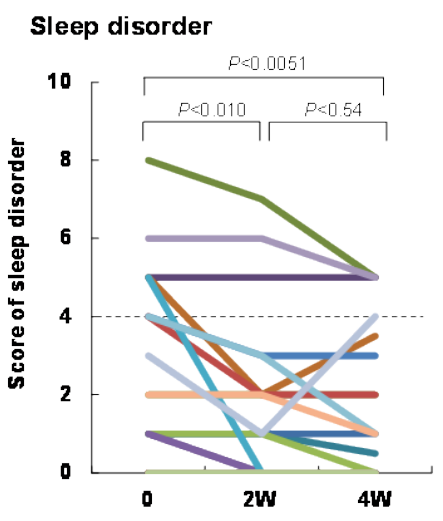

(a)

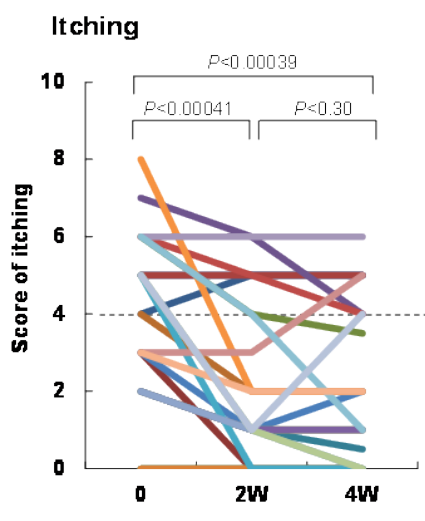

(b)

Figure 2. Effect of sacran solution on scores of sleep disorder (a) and itching (b) in patients with atopic dermatitis. Sacran solution was applied for 4 weeks in 25 patients with atopic dermatitis. After 2 and 4 weeks, the patients treated with sacran solution judged the score of sleep disorder and by themselves.

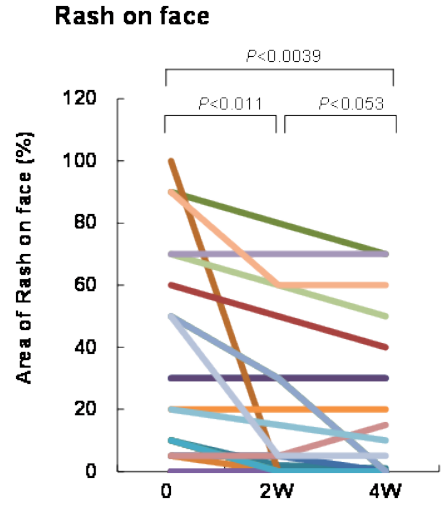

(a)

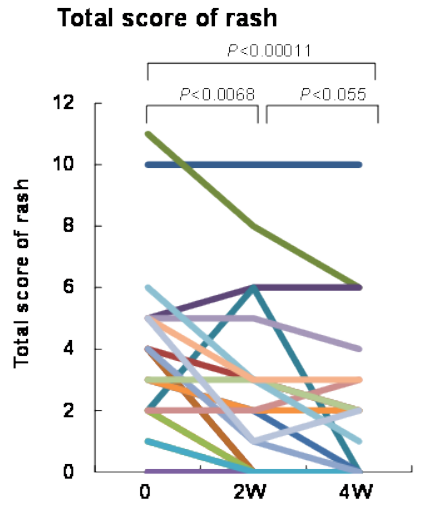

(b)

Figure 3. Effect of sacran solution on area of rash on face (a) and total score of rash (b) in patients with atopic dermatitis. Sacran solution was applied for 4 weeks in 25 patients with atopic dermatitis. After 2 and 4 weeks, the medical doctors diagnosed the rash of patients.

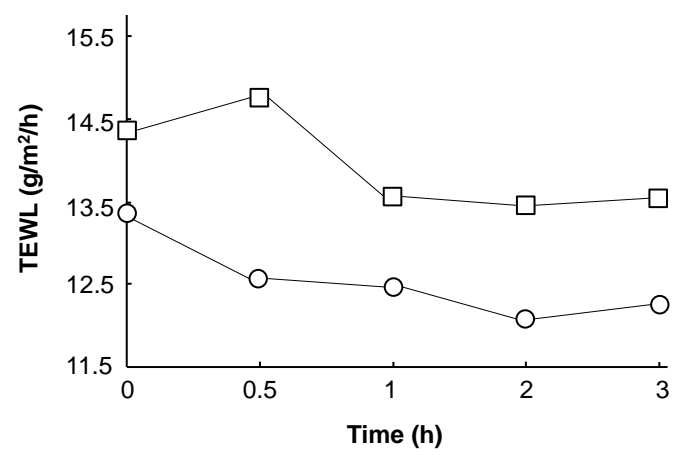

Figure 4. Effects of sacran solution on TEWL. TEWL was determined by a VapoMeter Wireless SWL4. Measurements will be undertaken after a $20 \mathrm{~min}$ rest period. Three acquisitions will be performed on the bony areas of the upper cheek of left and right sides. Each value represents the mean \pm S.E. of 10 experiments. 

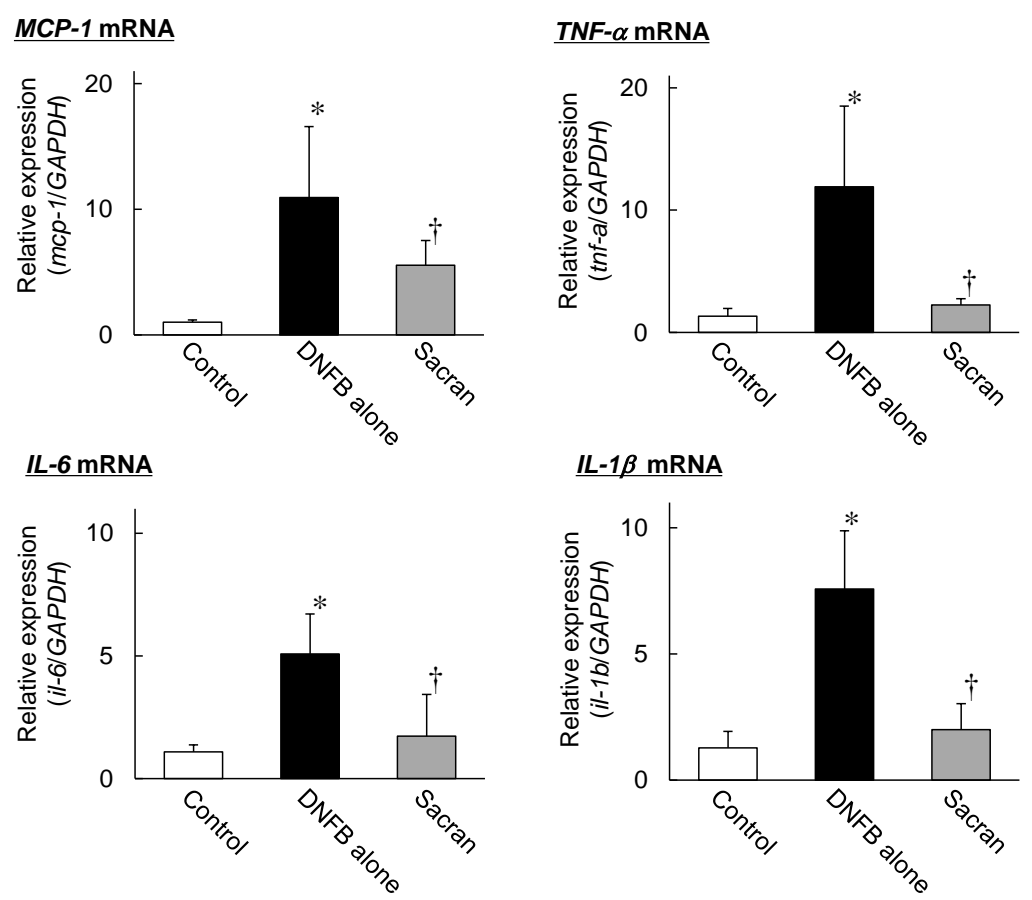

Figure 5. Effects of sacran on mRNA levels in immatured dermal skin model after treatment with sacran and DNFB for $24 \mathrm{~h}$. A three-dimensional cultured human skin, immatured dermal skin model was incubated with assay medium for $24 \mathrm{~h}$, and then sacran and DNFB were treated for $24 \mathrm{~h}$. The concentrations of sacran and DNFB were $0.05 \%$ and $0.01 \%$, respectively. After washing twice with PBS to remove the sample, total RNA was extracted by TRIzol ${ }^{\circledR}$ reagent and then the mRNA levels were determined by real-time PCR. Each value represents the mean \pm S.E. of 3 experiments. ${ }^{*} p<0.05$, compared with control. ${ }^{\dagger} p<0.05$, compared with DNFB alone.

whether a sacran can inhibit inflammatory responses in macrophages, we investigated the effect of sacran on the mRNA expression levels of TNF- $\alpha$ and COX-2 in RAW264.7 cells stimulated with PMA. The results showed that $0.05 \%(\mathrm{w} / \mathrm{v})$ sacran solution significantly suppressed the mRNA expression of TNF- $\alpha$ and COX-2 in macrophages, compared with PMA alone (Figure 6). These results indicate that sacran can reduce the inflammatory responses in macrophages.

\subsection{Inhibitory Effects of Sacran Solution on $\beta$-Hexosaminidase Release}

$\beta$-Hexosaminidase released from activated mast cells is responsible for the allergic inflammatory responses associated with disease states of atopic dermatitis. Therefore, to examine the anti-allergic activity of sacran, we evaluated the inhibitory effect of sacran on $\beta$-hexosaminidase release in RBL-2H3 cells, which is now known to be an analog of rat mucosal mast cells [22]. As shown in Figure 7, a sacran significantly lowered $\beta$-hexosaminidase release to $70 \%$, compared to control. These results suggest that a sacran can inhibit the allergic inflammatory responses.

The anti-inflammatory effects displayed by sacran, a unique cyanobacteria-derived glycosaminoglycanoid, could be related to the similarity of its chemical structure to that of glycosaminoglycans, sulfated polysaccharides, which possess numerous bioactivities including an anti-inflammatory effect [23]-[27]. The high sulfate and carboxyl group content of glycosaminoglycans permits them to interact with a wide range of proteins, enzymes, cytokines, chemokines, lipoproteins, and adhesion molecules [6]-[8] [28]. Also, Ngatu et al. reported that epicutaneous application of sacran to 2,4,6-trinitrochlorobenzene-induced NC/Nga mice significantly inhibited the development of allergic dermatitis skin lesions, and decreased the number of scratching behavior episodes by improving the stability, elasticity, and hydration of skin barrier as well as inhibiting the production of $\mathrm{T}_{\mathrm{H}} 2$ cytokines (IL-4, IL-5), $\mathrm{T}_{\mathrm{H}} 1$ cytokines (TNF- $\alpha$, IFN- $\gamma$ ) and inflammatory chemokines such as MCP-1 and 

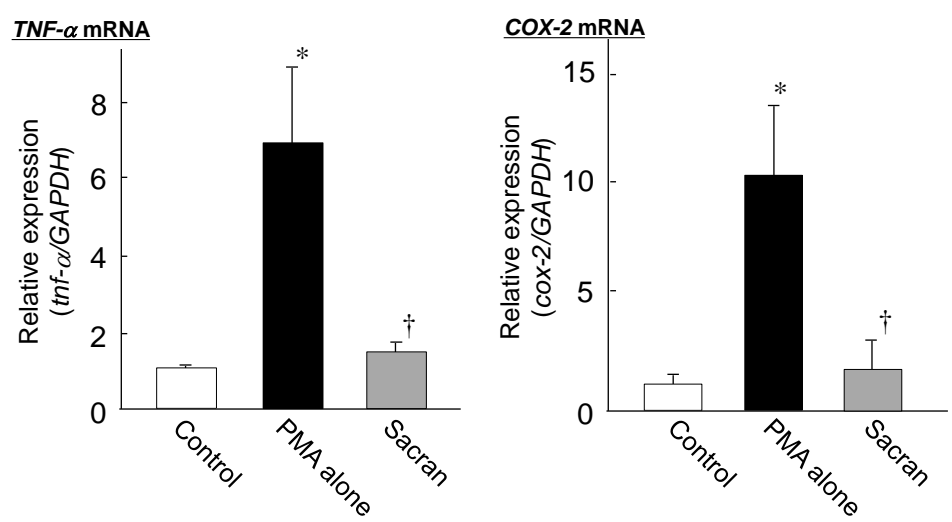

Figure 6. Effect of sacran on TNF- $\alpha$ and COX-2 mRNA Levels in RAW 264.7 cells stimulated with PMA. RAW 264.7 cells ( $5 \times 10^{5}$ cells/well) were incubated for $24 \mathrm{~h}$. Cells were washed once with PBS, and then incubated with $500 \mu \mathrm{L}$ of medium containing PMA in the presence or absence of sacran for $2 \mathrm{~h}$. After washing twice with, total RNA was extracted by TRIzol ${ }^{\circledR}$ reagent, and then the mRNA levels were determined by real-time PCR. Each value represents the mean \pm S.E. of $8-12$ experiments. ${ }^{*} p<0.05$, compared with control. ${ }^{\dagger} p<0.05$, compared with PMA alone.

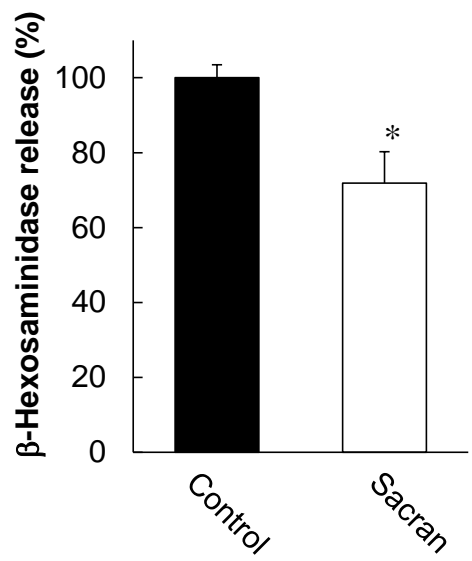

Figure 7. Effect of sacran on $\beta$-hexosaminidase release from RBL-2H3 cells. RBL-2H3 cells ( $2.5 \times 10^{5}$ cells/well) were incubated for $2 \mathrm{~h}$ with $500 \mu \mathrm{L}$ of anti DNP-IgE solution. After washing twice with MT buffer, the cells were incubated in the presence or absence of sacran for $10 \mathrm{~min}$. After adding $10 \mu \mathrm{L}$ of DNP-HSA solution, the cells were incubated for $30 \mathrm{~min}$. $\beta$-hexosaminidase release $(\%)=100 \times \mathrm{S} /(\mathrm{S}+\mathrm{CL}) \mathrm{S}$ : absorbance of supernatant. CL: absorbance of cell lysate. Each value represents the mean \pm S.E. of 3 experiments. ${ }^{*} p<0.05$, compared with control.

eotaxin; thus, inhibiting IgE and eosinophilic infiltration in mice [14]. These unique properties exhibited by sacran are not popular to most sulfated polysaccharides.

\section{Conclusion}

In the present study, we revealed that sacran solution has the potential to reduce atopic dermatitis in human, probably due to the suppression of inflammatory cytokine and chemokine mRNA levels in skin of atopic dermatitis. Sacran is a novel biomaterial and is applicable for skin care product for atopic dermatitis patients.

\section{Acknowledgements}

This study was supported financially by Daito Kasei Kogyo (Osaka, Japan). 


\section{References}

[1] Hengge, U.R., Ruzicka, T., Schwartz, R.A. and Cork, M.J. (2006) Adverse Effects of Topical Glucocorticosteroids. Journal of the American Academy of Dermatology, 54, 1-15. http://dx.doi.org/10.1016/j.jaad.2005.01.010

[2] Schoepe, S., Schacke, H., May, E. and Asadullah, K. (2006) Glucocorticoid Therapy-Induced Skin Atrophy. Experimental Dermatology, 15, 406-420. http://dx.doi.org/10.1111/j.0906-6705.2006.00435.x

[3] Ananthi, S., Raghavendran, H.R., Sunil, A.G., Gayathri, V., Ramakrishnan, G. and Vasanthi, H.R. (2010) In vitro Antioxidant and in Vivo Anti-Inflammatory Potential of Crude Polysaccharide from Turbinaria ornata (Marine Brown Alga). Food and Chemical Toxicology, 48, 187-192. http://dx.doi.org/10.1016/j.fct.2009.09.036

[4] Averbeck, M., Gebhardt, C.A., Voigt, S., Beilharz, S., Anderegg, U., Termeer, C.C., Sleeman, J.P. and Simon, J.C. (2007) Differential Regulation of Hyaluronan Metabolism in the Epidermal and Dermal Compartments of Human Skin by UVB Irradiation. Journal of Investigative Dermatology, 127, 687-697. http://dx.doi.org/10.1038/sj.jid.5700614

[5] Yanase, Y., Hiragun, T., Uchida, K., Ishii, K., Oomizu, S., Suzuki, H., Mihara, S., Iwamoto, K., Matsuo, H., Onishi, N., Kameyoshi, Y. and Hide, M. (2009) Peritoneal Injection of Fucoidan Suppresses the Increase of Plasma IgE Induced by OVA-Sensitization. Biochemical and Biophysical Research Communications, 387, 435-439. http://dx.doi.org/10.1016/j.bbrc.2009.07.031

[6] Bernfield, M., Gotte, M., Park, P.W., Reizes, O., Fitzgerald, M.L., Lincecum, J. and Zako, M. (1999) Functions of Cell Surface Heparan Sulfate Proteoglycans. Annual Review of Biochemistry, 68, 729-777. http://dx.doi.org/10.1146/annurev.biochem.68.1.729

[7] Kawashima, H., Atarashi, K., Hirose, M., Hirose, J., Yamada, S., Sugahara, K. and Miyasaka, M. (2002) Oversulfated Chondroitin/Dermatan Sulfates Containing GlcAb1/IdoAa1-3GalNAc (4,6-O-Disulfate) Interact with L- and P-Selectin and Chemokines. Journal of Biological Chemistry, 277, 12921-12930. http://dx.doi.org/10.1074/jbc.M200396200

[8] Taylor, K.R. and Gallo, R.L. (2006) Glycosaminoglycans and Their Proteoglycans: Host-Associated Molecular Patterns for Initiation and Modulation of Inflammation. FASEB Journal, 20, 9-22. http://dx.doi.org/10.1096/fj.05-4682rev

[9] Okajima, M.K., Bamba, T., Kaneso, Y., Hirata, K., Fukusaki, E., Kajiyama, S. and Kaneko, T. (2008) Supergiant Ampholytic Sugar Chains with Imbalanced Charge Ratio form Saline Ultra-Absorbent Hydrogels. Macromolecules, 41, 4061-4064. http://dx.doi.org/10.1021/ma800307w

[10] Okajima, M.K., Higashi, T., Asakawa, R., Mitsumata, T., Kaneko, D., Kaneko, T., Ogawa, T., Kurata, H. and Isoda, S. (2010) Gelation Behavior by the Lanthanoid Adsorption of the Cyanobacterial Extracellular Polysaccharide. Biomacromolecules, 11, 3172-3177. http://dx.doi.org/10.1021/bm101012u

[11] Okajima, M.K., Kaneko, D., Mitsumata, T., Kaneko, T. and Watanabe, J. (2009) Cyanobacteria That Produce Megamolecules with Efficient Self-Orientations. Macromolecules, 42, 3057-3062. http://dx.doi.org/10.1021/ma802829x

[12] Okajima, M.K., Miyazato, S. and Kaneko, T. (2009) Cyanobacterial Megamolecule Sacran Efficiently Forms LC Gels with Very Heavy Metal Ions. Langmuir, 25, 8526-8231. http://dx.doi.org/10.1021/la8036956

[13] Okajima, M.K., Nakamura, M., Mitsumata, T. and Kaneko, T. (2010) Cyanobacterial Polysaccharide Gels with Efficient Rare-Earth-Metal Sorption. Biomacromolecules, 11, 1773-1778. http://dx.doi.org/10.1021/bm100231q

[14] Ngatu, N.R., Okajima, M.K., Yokogawa, M., Hirota, R., Eitoku, M., Muzembo, B.A., Dumavibhat, N., Takaishi, M., Sano, S., Kaneko, T., Tanaka, T., Nakamura, H. and Suganuma, N. (2012) Anti-Inflammatory Effects of Sacran, a Novel Polysaccharide from Aphanothece sacrum, on 2,4,6-trinitrochlorobenzene-Induced Allergic Dermatitis in Vivo. Annals of Allergy, Asthma \& Immunology, 108, 117-122. http://dx.doi.org/10.1016/j.anai.2011.10.013

[15] Okajima, M.K., Ono, M., Kabata, K. and Kaneko, T. (2007) Extraction of Novel Sulfated Polysacchardes from Aphanothece sacrum, and Its Spectroscopic Characterization. Pure and Applied Chemistry, 79, 2039-2046.

[16] Bircher, A., de Boer, E.M., Agner, T., Wahlberg, J.E. and Serup, J. (1994) Guidelines for Measurement of Cutaneous Blood Flow by Laser Doppler Flowmetry. A Report from the Standardization Group of the European Society of Contact Dermatitis. Contact Dermatitis, 30, 65-72. http://dx.doi.org/10.1111/j.1600-0536.1994.tb00565.x

[17] Tewtrakul, S. and Subhadhirasakul, S. (2007) Anti-Allergic Activity of Some Selected Plants in the Zingiberaceae Family. Journal of Ethnopharmacology, 109, 535-538. http://dx.doi.org/10.1016/j.jep.2006.08.010

[18] Jin, H., He, R., Oyoshi, M. and Geha, R.S. (2009) Animal Models of Atopic Dermatitis. Journal of Investigative Dermatology, 129, 31-40. http://dx.doi.org/10.1038/jid.2008.106

[19] Gu, H., Huang, L., Wong, Y.P. and Burd, A. (2010) HA Modulation of Epidermal Morphogenesis in an Organotypic Keratinocyte-Fibroblast Co-Culture Model. Experimental Dermatology, 19, e336-e339. http://dx.doi.org/10.1111/j.1600-0625.2009.01052.x

[20] Homey, B., Steinhoff, M., Ruzicka, T. and Leung, D.Y. (2006) Cytokines and Chemokines Orchestrate Atopic Skin Inflammation. Journal of Allergy and Clinical Immunology, 118, 178-189. http://dx.doi.org/10.1016/j.jaci.2006.03.047

[21] Yamashita, H., Tasaki, D., Makino, T., Matsuoka, K., Nose, M., Inagaki, N. and Mizukami, H. (2009) The Role of IgE 
and Repeated Challenge in the Induction of Persistent Increases in Scratching Behavior in a Mouse Model of Allergic Dermatitis. European Journal of Pharmacology, 605, 153-157. http://dx.doi.org/10.1016/j.ejphar.2009.01.006

[22] Seldin, D.C., Adelman, S., Austen, K.F., Stevens, R.L., Hein, A., Caulfield, J.P. and Woodbury, R.G. (1985) Homology of the Rat Basophilic Leukemia Cell and the Rat Mucosal Mast Cell. Proceedings of the National Academy of Sciences of the United States of America, 82, 3871-3875. http://dx.doi.org/10.1073/pnas.82.11.3871

[23] Kuschert, G.S., Coulin, F., Power, C.A., Proudfoot, A.E., Hubbard, R.E., Hoogewerf, A.J. and Wells, T.N. (1999) Glycosaminoglycans Interact Selectively with Chemokines and Modulate Receptor Binding and Cellular Responses. Biochemistry, 38, 12959-12968. http://dx.doi.org/10.1021/bi990711d

[24] Parish, C.R. (2006) The Role of Heparan Sulphate in Inflammation. Nature Reviews Immunology, 6, 633-643. http://dx.doi.org/10.1038/nri1918

[25] Park, H.Y., Han, M.H., Park, C., Jin, C.Y., Kim, G.Y., Choi, I.W., Kim, N.D., Nam, T.J., Kwon, T.K. and Choi, Y.H. (2011) Anti-Inflammatory Effects of Fucoidan through Inhibition of NF-KappaB, MAPK and Akt Activation in Lipopolysaccharide-Induced BV2 Microglia Cells. Food and Chemical Toxicology, 49, 1745-1752. http://dx.doi.org/10.1016/j.fct.2011.04.020

[26] Volpi, N. (2006) Therapeutic Applications of Glycosaminoglycans. Current Medicinal Chemistry, 13, 1799-1810. http://dx.doi.org/10.2174/092986706777452470

[27] Yamada, S. and Sugahara, K. (2008) Potential Therapeutic Application of Chondroitin Sulfate/Dermatan Sulfate. Current Drug Discovery Technologies, 5, 289-301. http://dx.doi.org/10.2174/157016308786733564

[28] Salmivirta, M., Lidholt, K. and Lindahl, U. (1996) Heparan Sulfate: A Piece of Information. The FASEB Journal, 10, 1270-1279. 